\title{
Segmentation of Skin Lesions using Level Set Method
}

\author{
Zhen Ma, João Manuel R. S. Tavares \\ Instituto de Engenharia Mecânica e Gestão Industrial, \\ Faculdade de Engenharia, Universidade do Porto \\ Rua Dr. Roberto Frias, s/n, 4200-465 Porto - PORTUGAL
}

\begin{abstract}
Diagnosis of skin cancers with dermoscopy has been widely accepted as a clinical routine. However, the diagnostic accuracy using dermoscopy relies on the subjective judgment of the dermatologist. To solve this problem, a computer-aided diagnosis system is demanded. Here, we propose a level set method to fulfill the segmentation of skin lesions presented in dermoscopic images. The differences between normal skin and skin lesions in the color channels are combined to define the speed function, with which the evolving curve can be guided to reach the boundary of skin lesions. The proposed algorithm is robust against the influences of noise, hair, and skin textures, and provides a flexible way for segmentation. Numerical experiments demonstrated the effectiveness of the novel algorithm.
\end{abstract}

keywords: medical imaging, melanoma, image segmentation, level set method

\section{Introduction}

Nowadays, skin cancer has become one of the most frequent forms of cancer [1, 2]. An early diagnosis of skin cancer is critical for improving the prognosis, because patients with certain conditions, for example, the melanoma, can have a very high survival rate if the cancers are detected at the early stages and treated properly [3]. Dermoscopy is a non-invasive imaging technique developed to assist this diagnostic process, and has been reported to considerably improve the detection rate of skin cancers [4]. Nonetheless, it was also pointed out that the diagnostic accuracy using dermoscopy largely depends on dermatologists' experience [5]. In order to eliminate this subjectivity, a computer-aided diagnosis (CAD) system is demanded.

The first step of a CAD system is to segment skin lesions in the images; the accuracy of segmentation has a deterministic influence on the later analysis. The appearance of skin lesions varies considerably among different skin conditions; meanwhile, the influences of noise, hairs, skin texture, and air bubbles may appear simultaneously in the image and make the segmentation even harder. Many algorithms have been proposed to solve the segmentation problem, and the majority of them are based on thresholding and clustering. For example, a double thresholding process was used in [6] to segment the boundaries of skin lesions based on the intensity of the converted images. A dermatologist-like tumor extraction algorithm and its improved version were developed in $[7,8]$ that combined the thresholding with the iterative region 
growing for segmentation. A 2D color clustering algorithm was proposed in [9]; a supervised algorithm based on a neural network and an unsupervised algorithm based on modified JSEG algorithm were proposed in [10] and [11], respectively.

The level set method was initially developed to track curve evolution in computational physics; however, it has been successfully applied to many areas of image processing [12]. For the segmentation of dermoscopic images, the level set method is less sensitive to the influence of noise; and the implicit tracking provides an efficient way to obtain the boundary and the regions of skin lesions simultaneously. Here, a new algorithm based on the level set method was proposed to fulfil the segmentation task. Following the statistical features of dermoscopic images in different color spaces, the contrasts of the lightness and saturation between the skin lesions and the surrounding normal skin were used as the clues for segmentation and were combined to define a region-based external force, following which the evolving curve can contract to the boundary of the skin lesion in a robust way.

In the next section, the level set method is reviewed; then, the proposed algorithm is introduced, including the equation of motion and the evolution process; afterwards, numerical experiments are presented, and according to the segmentation results, implementation issues of the algorithm are discussed. In the last section, the conclusions and perspectives of future work are indicated.

\section{Methodology}

The level set method was proposed to solve the topological changes during the curve evolution [13]. In this method, the evolving curve is embedded into a higherdimensional level set function $\phi(x, y, t)$ as its zero level set, and the evolution is tracked by finding the zero level set of the function $\phi(x, y, t)$ at the time $t$. The equation of motion of a level set method is normally written as:

$\frac{\partial \phi}{\partial t}+F|\nabla \phi|=0$,

where $\phi(x, y, t)$ is the level set function and $F$ is the speed function. The main idea of using the level set method for segmentation is to model the segmentation as a process of curve evolution. Hence, a proper speed function needs to be defined, with which the curve can reach the object boundary and achieve a stable status there.

\subsection{Equation of motion}

The color distribution of skin lesions is normally inhomogeneous. If the curve evolves inside the region of skin lesions, it can be easily attracted to the inner boundaries and cause wrong segmentation. Therefore, in the proposed algorithm, the curve evolution is constrained to contraction in the region of normal skin. By this way, the initial curves are required to cover the entire regions of the skin lesions. The values of the level set function $\phi(x, y, 0)$ are then defined as the signed distance function to the initial curves with positive (negative) sign inside (outside) the curves. 
The lightness difference between normal skin and skin lesions provides an important clue for segmentation. Nevertheless, the appearance of skin lesions has large variations among different conditions, and in many cases, its main dissimilarity to the normal skin is the chromaticity which is often perceptually affected by lightness variations. Thus, in order to use the color information efficiently, the RGB color space in the images are converted to the CIE $L^{*} a^{*} b^{*}$ and CIE $L^{*} u^{*} v^{*}$ color spaces. Although the lightness is separated from the color representation in the two CIE color spaces, the chromaticity channels $a^{*}, b^{*}, u^{*}$ and $v^{*}$ are coordinates in the color diagram and are unsuitable to be used directly to define the speed function. Instead, the color saturation was adopted to combine the lightness and chromaticity for segmentation. Saturation is a measure that describes the colorfulness of a color relative to its lightness, but is not officially defined in the CIE color system. The definition of saturation in computer vision was adopted here with its value calculated as:

$S=\left\{\begin{array}{cl}0 & \text { if } R+G+B=0 \\ 1-\frac{\min (R, G, B)}{(R+G+B) / 3} & \text { otherwise }\end{array}\right.$.

Then, the equation of motion of the proposed level set model is defined as:

$\frac{\partial \phi}{\partial t}+P_{L}(x, y) * P_{S}(x, y) *(1+\kappa)|\nabla \phi|=0$,

where $\kappa$ is the curve curvature; $P_{L}(x, y)$ and $P_{S}(x, y)$ are the Gaussian probability density distribution function of the lightness and saturation channels of the normal skin, respectively. The curvature defined in the speed function acts as the internal force to smooth the curve during the evolution.

The speed function in Eq. (3) includes the statistical information of lightness and saturation values of normal skin. However, this information is unavailable before segmentation; hence, to obtain an approximation of these values, the Otsu's method [14] is applied to classify the image pixels based on the lightness values. Supposing $\Omega_{0}$ is the set composed by pixels that represent the normal skin according to the classification of the Otsu's method, the following region is used to calculate the statistical values:

$\Omega_{S}=\{(x, y) \mid-50<\phi(x, y, 0)<0\} \cap \Omega_{0}$.

The region $\Omega_{S}$ belongs to a neighboring external band of the initial curves; as the skin lesions are completely inside the initial curves, this region can provide an approximation of the statistical distributions of the lightness and saturation of the normal skin. These statistical values are then updated along with the curve evolution. With Eq. (3), the evolving curves will contract to the places where either the lightness or the saturation is appreciably different to normal skin.

\subsection{Evolution}

As referred before, the $a^{*}, b^{*} u^{*}, v^{*}$ channels in the CIE $L^{*} a^{*} b^{*}$ and $L^{*} u^{*} v^{*}$ color spaces are the positions of a color relative to the color bases and diagram. Their loca- 
tions reflect the perceptual difference between the normal skin and skin lesions; pixels of the same group should have coordinates near each other, and pixels from the different group should have coordinates with a large distance. Accordingly, the image pixels can be classified into two groups based on their distances to the spatial color centers of normal skin and skin lesions. Nonetheless, the spatial centers of the two groups are unknown either. Yet, given that the skin lesions are inside the evolving curves, a neighboring external region $\Omega_{S}$ is used to calculate the spatial centers of normal skin in the color space as:

$\Omega_{S}^{(t)}=\{(x, y) \mid-50<\phi(x, y, t)<0\}$,

and the internal region of the curve is used to calculate the centers of the skin lesions. Along with the contraction of the curve, the spatial centers of the skin lesions and the surrounding normal skin will become more accurate. With the classification based on the Euclidean distance in the color spaces, the statistical distribution of the saturation values of normal skin can be better reflected. Hence, the statistical vales are updated during the evolution in the region $\Omega_{0}^{(t)} \cap \Omega_{S}^{(t)}$ where $\Omega_{0}^{(t)}$ is the set composed by pixels representing the normal skin at the time $t$. Additionally, in light with the color classification, the speed function in Eq. (3) is modified as:

$F^{*}(x, y)=\left\{\begin{array}{cl}0.5 * F(x, y) & \text { if }(x, y) \text { represents skin lesions at the time } t \\ F(x, y) & \text { otherwise }\end{array}\right.$.

With the modified speed function, the evolving curve can be further attached to the boundary of skin lesions.

\section{Experiments}

An image database containing 68 dermoscopic images was used to test the performance of the proposed segmentation algorithm, in which 58 were diagnosed as nevus and 10 as melanomas. CUDA implementation of the proposed algorithm was adopted to enhance the computational efficiency. The obtained segmentation results were quite promising and, for their quantitative analysis, the exclusive-or measure defined below was used to evaluate the difference between the ground truth and the segmentation result:

$D\left(C_{0}, C_{1}\right)=\operatorname{Area}\left(\operatorname{inside}\left(C_{0}\right) \oplus \operatorname{inside}\left(C_{1}\right)\right) / \operatorname{Area}\left(\operatorname{inside}\left(C_{0}\right)\right)$,

where $C_{0}$ is the ground true boundary, $C_{1}$ is the contour obtained by the algorithm, and $\bigoplus$ is the exclusive-or operator. Fig. 1 illustrates four segmentation examples in the image database; one can verify the robustness of the proposed approach against the different imaging conditions. For the proposed algorithm, the mean and the standard deviation of the exclusive-or measure on this image database are 0.1036 and 0.0485 , respectively.

There is no restrict on the shape of the initial curves in the proposed algorithm; however, the initial curves are required to cover the complete region of the skin le- 
sions. If the neighboring region of the initial curves is affected appreciably by unwanted influences, the algorithm may not achieve satisfactory results; to avoid this situation, the initial curves are defined manually. Meanwhile, in the segmentation, the size of the neighboring region of the evolving curves affects the statistical information of normal skin around the skin lesions. A larger neighboring region around the initial curve can capture the variations of normal skin more accurately, but is more likely to introduce unwanted influences. The band size was chosen as 50 in the experiments and led to satisfactory results.

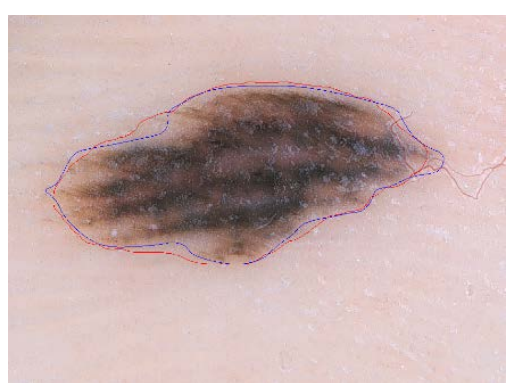

(a)

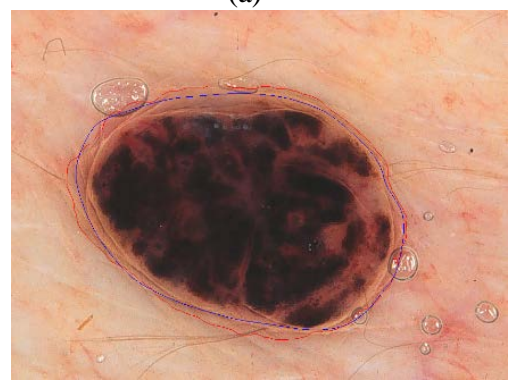

(c)

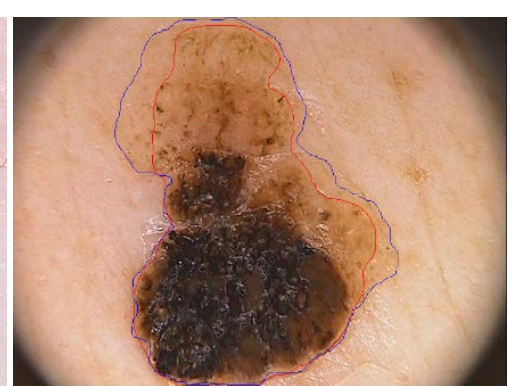

(b)

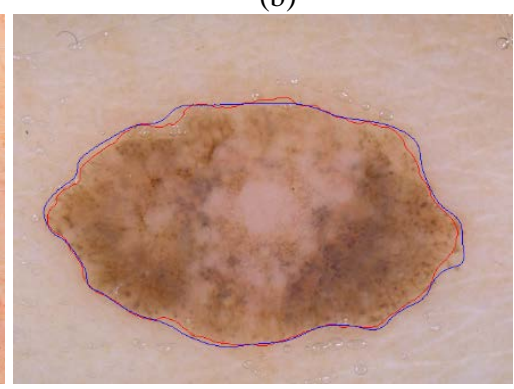

(d)

Fig. 1 Segmentation examples using the proposed algorithm, red contours - segmentation results of the proposed algorithm; blue contours - ground truths: (a) image with nevi, $D\left(C_{0}, C_{1}\right)=0.0833$; (b) image with melonoma, $D\left(C_{0}, C_{1}\right)=0.1590$; (c) image with nevi, $D\left(C_{0}, C_{1}\right)=0.0868$; (d) image with melanoma, $D\left(C_{0}, C_{1}\right)=0.0447$.

\section{CONCLUSION}

A novel level set method was proposed to segment skin lesions. The proposed algorithm combines the various information contained in dermoscopic images, and defines the speed function based on the converted color channels. Numerical experiments illustrated the effectiveness and robustness of the algorithm, and the implementation issues were discussed based on the tests.

The differences between the skin lesions and normal skin were efficiently used in the proposed algorithm. With the region-based external forces, the proposed algorithm is not sensitive to the unwanted influences presented in the images. The future work will continue to improve its robustness and accuracy. 


\section{ACKNOWLEDGEMENT}

This work was done in the scope of the project "A novel framework for supervised mobile assessment and risk triage of skin lesions via non-invasive screening", with the reference PTDC/BBB-BMD/3088/2012, financially supported by Fundação para a Ciência e a Tecnologia (FCT), in Portugal.

\section{REFERENCES}

1. Jemal A, Siegel R, Xu J, Ward E. Cancer statistics, 2010. CA Cancer J Clin. 2010; 60(5):288-296.

2. American Cancer Society. Cancer facts \& figures 2013. http://www.cancer.org/acs/groups/content/@epidemiologysurveilance/documents/docume nt/acspc-036845.pdf.

3. Balch CM, Buzaid AC, Soong SJ, Atkins MB, Cascinelli N, Coit DG, Fleming ID, Gershenwald JE, Houghton A Jr, Kirkwood JM, McMasters KM, Mihm MF, Morton DL, Reintgen DS, Ross MI, Sober A, Thompson JA, Thompson JF. Final version of the American Joint Committee on Cancer staging system for cutaneous melanoma. J Clin Oncol. 2001; 19(16):3635-3648.

4. Grin CM, Kopf AW, Welkovich B, Bart RS, Levenstein MJ. Accuracy in the clinical diagnosis of malignant melanoma. Arch Dermatol. 1990; 126(6):763-766.

5. Kittler H, Pehamberger H, Wolff K, Binder M. Diagnostic accuracy of dermoscopy. Lancet Oncol. 2002; 3(3):159-165.

6. Xu L, Jackowski M, Goshtasby A, Roseman D, Bines S, Yu C, Dhawan A, Huntley A. Segmentation of skin cancer images. Image Vision Comput. 1999; 17(1):65-74.

7. Iyatomi H, Oka H, Saito M, Miyake A, Kimoto M, Yamagami J, Kobayashi S, Tanikawa A, Hagiwara M, Ogawa K, Argenziano G, Soyer HP, Tanaka M. Quantitative assessment of tumor extraction from dermoscopy images and evaluation of computer-based extraction methods for automatic melanoma diagnostic system. Melanoma Res. 2006; 16(2):183-190.

8. Iyatomi H, Oka H, Celebi ME, Hashimoto M, Hagiwara M, Tanaka M, Ogawa K. An improved Internet-based melanoma screening system with dermatologist-like tumor area extraction algorithm. Comput Med Imaging Graph. 2008; 32(7):566-579.

9. Schmid P. Segmentation of digitized dermatoscopic images by two-dimensional color clustering. IEEE T Med Imaging. 1999; 18(2):164-171.

10. Donadey T, Serruys C, Giron A, Aitken G, Vignali J-P, Triller R, Fertil B. Boundary detection of black skin tumors using an adaptive radial-based approach. SPIE Med Imaging. 2000; 3379:810-816.

11. Celebi ME, Aslandogan YA, Stoecker WV, Iyatomi H, Oka H, Chen X. Unsupervised border detection in dermoscopy images. Skin Res Technol. 2007; 13(4):454-462.

12. Ma Z, Tavares JMRS, Jorge RNM, Mascarenhas T. A review of algorithms for medical image segmentation and their applications to the female pelvic cavity. Comput Methods Biomech Biomed Engin. 2010; 13(2): 235-246.

13. Osher SJ, Sethian JA. Fronts propagating with curvature-dependent speed: algorithms based on Hamilton-Jacobi formulations. J Comput Phys. 1998; 79(1):12-49. 
14. Otsu N. A threshold selection method from gray-level histograms". IEEE T Syst Man Cyb. 1979; 9(1):62-66. 